\title{
Prognostic Factors and Survival Outcomes of Lung Cancer Patients with Brain Metastasis: Experience of a Tertiary Cancer Centre
}

Tibdewal AR, Pathak RS, Laskar SG and Agarwal JP*

Department of Radiation Oncology, Tata Memorial Centre, Mumbai, India

\begin{abstract}
Objectives : Lung cancer is one of the leading causes of cancer deaths in India. Fifteen to $35 \%$ of patients present with Brain Metastasis (BM) and are treated with palliative Whole Brain Radiotherapy (WBRT). We report the survival outcomes and prognostic factors of lung cancer patients with BM.

Methods: Two hundred and twenty-one patients were analysed from July 2010 - June 2014 who received palliative WBRT. Overall Survival (OS) was computed using Kaplan Meier method. Difference in survival for known prognostic factors were analysed using log rank test with significance of $p$ value at 0.05 and $95 \%$ confidence interval.

Results: Median OS was 3.7 months. OS at 6, 12 and 24 months was $36 \%, 24 \%$ and $13.8 \%$ respectively. Synchronous BM patients had significantly better OS compared to those with metachronous BM with a hazard ratio (HR) $0.62(95 \% \mathrm{Cl}$ $0.46-0.85, p=0.01$ ). Patients with a higher Graded Prognostic Assessment (GPA) score, a Recursive Partitioning Analysis (RPA) class of I-II and adenocarcinoma histology had comparatively better OS. There was a subset of patients $(n=58)$ who died within 30 days of diagnosis of BM. Neither RPA class nor GPA score could accurately predict patients who were within the 30 day mortality group.
\end{abstract}

Conclusion: Patients of lung cancer with BM have poor outcome. GPA score of 3.5-4.0, RPA class I-II and adenocarcinoma histology showed better survival outcomes. However, neither GPA nor RPA could predict 30 day mortality.

Keywords: Brain metastasis; Lung cancer; Prognostic factors; 30 day mortality; RPA class; GPA score

\section{Introduction}

Brain metastases (BM) are a major cause of morbidity and mortality in patients with lung cancer. Treatment options for BM include surgery, stereotactic radiosurgery (SRS), whole brain radiotherapy (WBRT) and supportive care. Median survival of patients with BM is approximately 4-7 months [1,2]. A significant number of patients of lung cancer with $\mathrm{BM}$ are not eligible for any treatment and are offered best supportive care alone.

Various prognostic scores are available that aim at classifying patients with $\mathrm{BM}$ into different prognostic groups like Recursive partitioning analysis (RPA), Graded Prognostic Assessment (GPA) and Mini Mental Status Examination (MMSE). RPA classifies patients into 3 prognostic groups based on age, Karnofsky Performance Score (KPS), status of primary disease and Extra Cranial Metastases (ECM). In addition to the above, GPA includes the number of brain lesions also as a prognostic factor. Time of presentation of BM has been found to impact the outcome of the patient where metachronous BM have better outcome compared to synchronous BM $[3,4]$. There are various other biological and molecular markers that are considered prognostically important. However, they haven't found a place in routine clinical practice yet.

The aim of this retrospective review is to report survival outcomes and prognostic factors in patients of lung cancer with BM as well as to validate the GPA scoring in Indian population.

\section{Materials and Methods}

Two hundred and twenty one consecutive patients of lung cancer with BM treated in department of radiation oncology of Tata Memorial Centre, India from July 2010 to June 2014 were eligible for this study. Detailed inclusion criteria and WBRT technique are given in our previous study report [5]. Follow up data was collected and updated from the case files, electronic medical records and by telephonic contact whenever necessary.

\section{Statistical Analysis}

The primary objective of this study was to report the survival outcomes according to RPA class, GPA scores and to validate the GPA scoring system in our population. Overall survival (OS) was calculated from the date of diagnosis of BM until the date of death from any cause or last known follow up date. Survival analysis was computed using Kaplan Meier method and comparisons were made using Log Rank test. Two-sided p-values were considered significant if less than 0.05 . Prognostic factors were evaluated first with univariate analysis and those found significant were tested with multivariate cox proportional hazard model. Univariate tests were not adjusted for multiple comparisons. Statistical analysis was performed with SPSS software for Windows, version 21.0 (SPSS, Chicago, IL, USA).

\section{Results}

\section{Patients and treatment characteristics}

The baseline patients and tumor characteristics are presented in

*Corresponding author: Agarwal JP, Department of Radiation Oncology Tata Memorial Centre, Mumbai-400012, India, Tel: 91-22-24177164; E-mail agarwaljp@tmc.gov.in

Received January 19, 2016; Accepted March 12, 2016; Published March 21 2016

Citation: Tibdewal AR, Pathak RS, Laskar SG, Agarwal JP (2016) Prognostic Factors and Survival Outcomes of Lung Cancer Patients with Brain Metastasis: Experience of a Tertiary Cancer Centre. J Brain Tumors Neurooncol 1: 106. doi: 10.4172/2475-3203.1000106

Copyright: (c) 2016 Tibdewal AR, et al. This is an open-access article distributed under the terms of the Creative Commons Attribution License, which permits unrestricted use, distribution, and reproduction in any medium, provided the original author and source are credited. 
Citation: Tibdewal AR, Pathak RS, Laskar SG, Agarwal JP (2016) Prognostic Factors and Survival Outcomes of Lung Cancer Patients with Brain Metastasis: Experience of a Tertiary Cancer Centre. J Brain Tumors Neurooncol 1: 106. doi: 10.4172/2475-3203.1000106

Page 2 of 5

Tables 1 and 2. Of the 221 patients included in the study, 147 (66.5\%) were males and $74(33.5 \%)$ were females with median age of 55 years (range from 2288 years). Majority of our patients $(54.3 \%)$ had T4 tumors of adenocarcinoma histology. Most of the patients $(57.5 \%)$ had extracranial metastasis at the time of diagnosis of BM. Treatment related details are given in Table 3. Treatment strategies were designed to suit the type of patient population commonly encountered. Since a large number of patients had poor KPS with advance stage lung cancer and extra cranial metastasis, they were treated with WBRT alone. The most common dose fractionation regimen used was $20 \mathrm{~Gy}$ delivered in 5 fractions (87.1\%). Though systemic therapy forms an integral part of patient management for metastatic lung cancer, only $59.3 \%$ of them received it.

\section{Outcomes}

At the time of analysis, only $31 / 221$ patients (14\%) were alive. Median OS for the entire patient population from the date of diagnosis of BM was 3.7 months (95\% CI 2.6-4.8 months; Figures 1-3). Overall survival at 6,12 and 24 months was $36 \%, 24 \%$ and $13.8 \%$ respectively. Twenty six patients (11.8\%) were lost to follow up. Median OS according to presentation of BM, RPA class and GPA score are given in Table 4 . Majority of the patients (215/221) belonged to RPA class II or III. Similarly most of the patients (199/221) had a GPA score range of 0 to 2.5. Patients with the most favourable RPA class, GPA score, KPS of the patient at diagnosis of BM and status of primary lung cancer had a significantly longer median OS compared to any other patient. Out of 221 patients, 57 (25.8\%) did not survive even for 30 days. In this ' 30 day mortality group' of patients, $38 / 57$ (67\%) belonged to RPA class III and $49 / 57$ (86\%) patients had a GPA score of $\leq 2.5$.

\begin{tabular}{|c|c|}
\hline Variables & $\mathrm{N}=221(\%)$ \\
\hline \multicolumn{2}{|l|}{ Age } \\
\hline$<50$ & $74(33.5)$ \\
\hline $50-59$ & $79(35.7)$ \\
\hline$\geq 60$ & $68(30.8)$ \\
\hline \multicolumn{2}{|l|}{ Gender } \\
\hline Male & $147(66.5)$ \\
\hline Female & 74 (33.5) \\
\hline \multicolumn{2}{|l|}{ KPS } \\
\hline$<70$ & $106(48)$ \\
\hline $70-80$ & $99(44.8)$ \\
\hline $90-100$ & $16(7.2)$ \\
\hline \multicolumn{2}{|l|}{ Presentation } \\
\hline Synchronous & $128(57.9)$ \\
\hline Metachronous & $93(42.1)$ \\
\hline \multicolumn{2}{|l|}{ RPA } \\
\hline 1 & $06(2.7)$ \\
\hline II & $109(49.3)$ \\
\hline III & $106(48)$ \\
\hline \multicolumn{2}{|l|}{ GPA } \\
\hline $0-1$ & $88(39.8)$ \\
\hline $1.5-2.5$ & $111(50.2)$ \\
\hline 3 & $17(7.7)$ \\
\hline $3.5-4$ & $05(2.3)$ \\
\hline \multicolumn{2}{|l|}{ Status } \\
\hline Alive & $31(14)$ \\
\hline Dead & $164(74.2)$ \\
\hline Unknown & $26(11.8)$ \\
\hline
\end{tabular}

Table 1: Patient characteristics

\begin{tabular}{|c|c|}
\hline Variables & $\mathbf{N}(\%)$ \\
\hline \multicolumn{2}{|l|}{ Tumor stage } \\
\hline $\mathrm{T} 1-\mathrm{T} 2$ & $47(21.3)$ \\
\hline T3 & $54(24.4)$ \\
\hline T4 & $120(54.3)$ \\
\hline \multicolumn{2}{|l|}{ Nodal stage } \\
\hline No & $28(12.7)$ \\
\hline N1 & $14(6.3)$ \\
\hline N2 & $101(45.7)$ \\
\hline N3 & $73(33.0)$ \\
\hline \multicolumn{2}{|l|}{ Histology } \\
\hline Adenocarcinoma & $165(74.7)$ \\
\hline Non adenocarcinomas & $56(25.3)$ \\
\hline \multicolumn{2}{|l|}{ No of Mets } \\
\hline 1 & $54(24.4)$ \\
\hline 3-Feb & $41(18.6)$ \\
\hline$>3$ & $92(41.6)$ \\
\hline Unknown & $34(15.4)$ \\
\hline \multicolumn{2}{|l|}{ Extra cranial Metastases } \\
\hline Yes & $127(57.5)$ \\
\hline No & $89(40.3)$ \\
\hline Unknown & $05(2.3)$ \\
\hline
\end{tabular}

Table 2: Tumor Characteristics.

\begin{tabular}{|c|c|c|}
\hline Treatment & Characteristics & N (\%) \\
\hline \multirow{2}{*}{ Treatment Modality } & WBRT alone & $209(94.6)$ \\
\cline { 2 - 3 } & Surgery $\rightarrow$ PORT & $12(5.4)$ \\
\hline \multirow{3}{*}{ Radiation Dose Fractionation } & $12 \mathrm{~Gy} / 2 \mathrm{Fr}$ & $10(4.5)$ \\
\cline { 2 - 3 } & $20 \mathrm{~Gy} / 5 \mathrm{Fr}$ & $192(87.1)$ \\
\cline { 2 - 3 } & $30-39 \mathrm{~Gy} / 10-13 \mathrm{Fr}$ & $19(8.4)$ \\
\hline \multirow{2}{*}{ Systemic therapy } & Yes & $131(59.3)$ \\
\cline { 2 - 3 } & No & $42(19.0)$ \\
\cline { 2 - 3 } & Unknown & $48(21.7)$ \\
\hline
\end{tabular}

WBRT-Whole Brain Radiotherapy; PORT-Post-Operative Radiotherapy; FrFraction

Table 3: Treatment characteristics for BM.

\section{Prognostic factors}

Numerous variables have been evaluated using univariate analysis for their prognostic significance as shown in Table 4. Presentation of BM, KPS, histology, RPA class, GPA score, and controlled primary could significantly determine OS as shown in Table 4. However, age, number of brain lesion and ECM were not significant. Multivariate analysis as shown in Table 5 revealed that adenocarcinoma histology, synchronous presentation of BM, RPA class I and II, and GPA score of 3.5-4.0 were independent positive prognostic factor of OS.

\section{Discussion}

Median OS of the present study population was in concurrence with the existing outcomes reported in the literature as shown in Table 6 [6,7]. However, outcomes have to be compared with caution as present study was not restricted to non-small cell histology as in other studies. Primary tumor was controlled in only $8.6 \%$ of patients in the present study as compared to $67 \%$ of those in RTOG study [7]. This difference could be because we included consecutive unselected patient population for this retrospective study. Inclusion of consecutive patients, gives a true reflection of the cases encountered in routine clinics and may be relevant for developing countries that have similar patient population and treatment strategies. 
Citation: Tibdewal AR, Pathak RS, Laskar SG, Agarwal JP (2016) Prognostic Factors and Survival Outcomes of Lung Cancer Patients with Brain Metastasis: Experience of a Tertiary Cancer Centre. J Brain Tumors Neurooncol 1: 106. doi: 10.4172/2475-3203.1000106

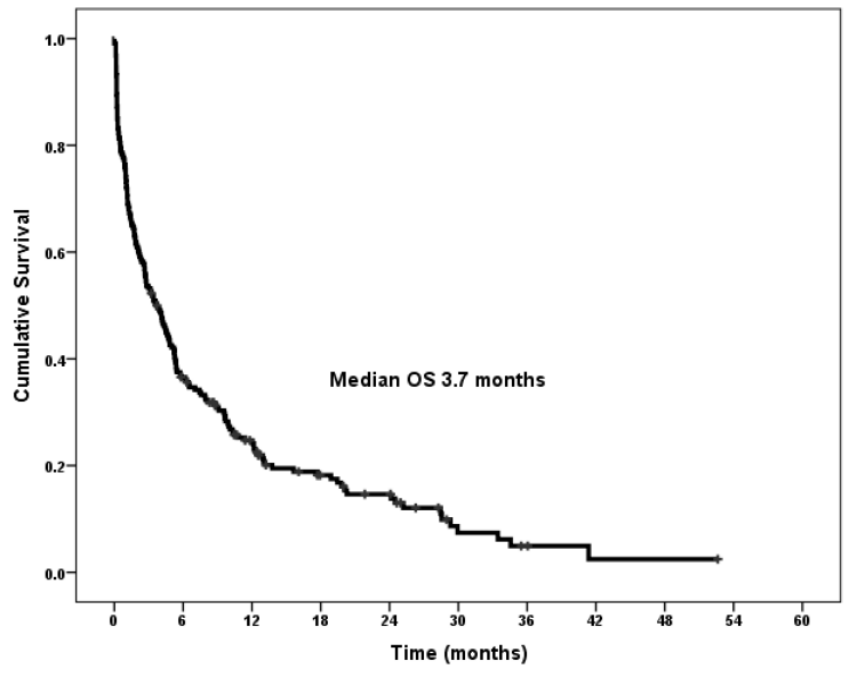

Figure 1: Kaplan-Meier curve depicting overall survival (OS) curve.

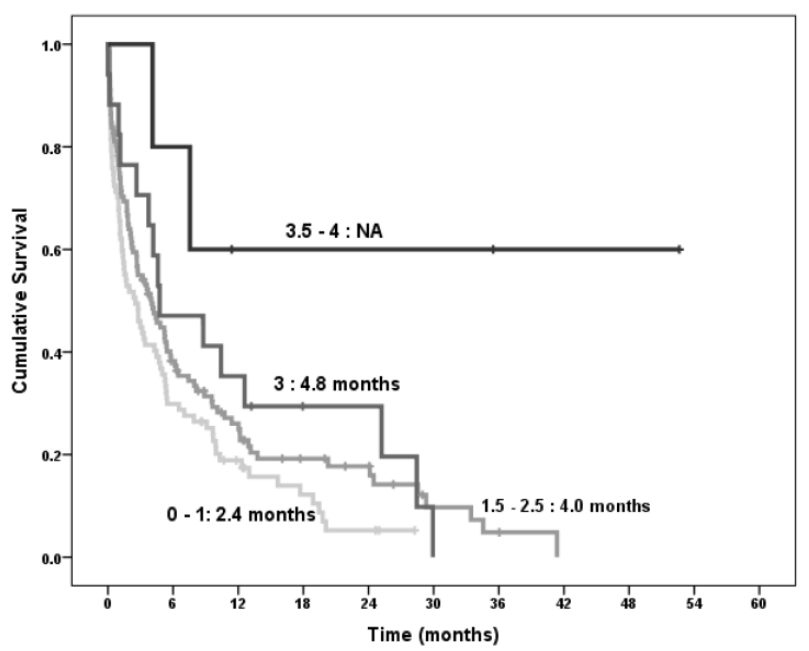

Figure 2: Kaplan-Meier curve for overall survival (OS) stratified by graded prognostic assessment (GPA) score.

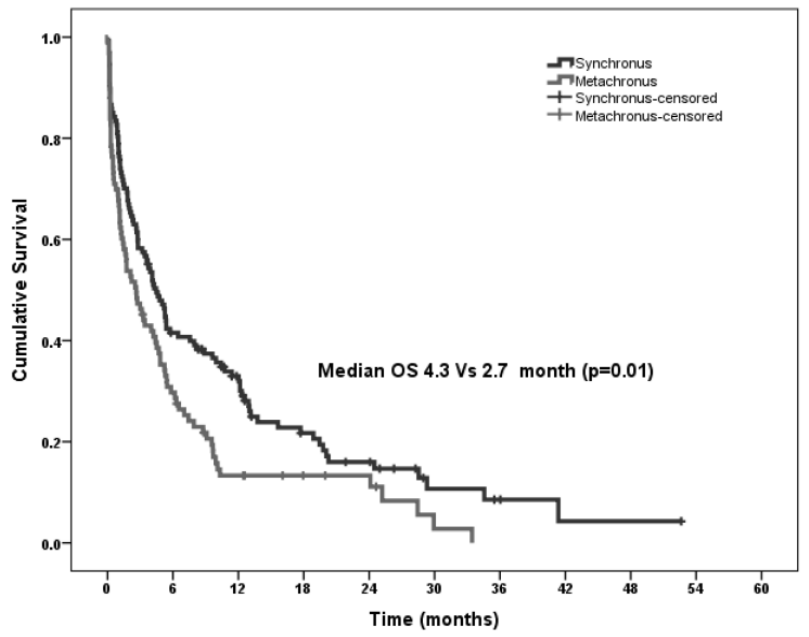

Figure 3: Kaplan-Meier curve for overall survival (OS) stratified by presentation of BM. 
Citation: Tibdewal AR, Pathak RS, Laskar SG, Agarwal JP (2016) Prognostic Factors and Survival Outcomes of Lung Cancer Patients with Brain Metastasis: Experience of a Tertiary Cancer Centre. J Brain Tumors Neurooncol 1: 106. doi: 10.4172/2475-3203.1000106

Page 4 of 5

\begin{tabular}{|c|c|c|}
\hline Variable (N) & Median OS in months $(95 \% \mathrm{Cl})$ & p-Value \\
\hline \multicolumn{2}{|l|}{ Age } & \multirow{4}{*}{0.11} \\
\hline$>60(68)$ & $2.7(1.0-4.3)$ & \\
\hline $50-59(79)$ & $4.5(2.0-2.0)$ & \\
\hline$<50(74)$ & $3.9(2.4-5.4)$ & \\
\hline \multicolumn{2}{|l|}{ Gender } & \multirow{3}{*}{0.19} \\
\hline Male (147) & $3.1(2.0-4.3)$ & \\
\hline Female (74) & $4.3(2.8-5.7)$ & \\
\hline \multicolumn{2}{|l|}{ KPS } & \multirow{4}{*}{0} \\
\hline$<70(106)$ & $2.8(1.4-4.2)$ & \\
\hline $70-80$ (99) & $4.1(1.7-6.5)$ & \\
\hline $90-100(16)$ & $12.6(0.0-34.5)$ & \\
\hline \multicolumn{2}{|l|}{ Presentation } & \multirow{3}{*}{0.01} \\
\hline Synchronous (128) & $4.3(3.0-5.6)$ & \\
\hline Metachronous (93) & $2.7(1.3-4.0)$ & \\
\hline \multicolumn{2}{|l|}{ Histology } & \multirow{3}{*}{0.01} \\
\hline Adenocarcinoma (165) & $4.4(2.6-6.2)$ & \\
\hline Non-Adenocarcinoma (56) & $2.6(1.5-3.7)$ & \\
\hline \multicolumn{2}{|l|}{ RPA Class } & \multirow{4}{*}{0.02} \\
\hline I (06) & $8.8(0.0-27.4)$ & \\
\hline II (109) & $5.2(2.3-8.1)$ & \\
\hline III (106) & $2.6(1.0-4.2)$ & \\
\hline \multicolumn{2}{|l|}{ GPA Score } & \multirow{5}{*}{0.01} \\
\hline 0-1 (88) & $2.4(1.0-3.7)$ & \\
\hline $1.5-2.5(111)$ & $4.0(2.2-5.8)$ & \\
\hline $3(17)$ & $4.8(0.0-11.0)$ & \\
\hline $3.5-4(05)$ & NR (NA) & \\
\hline \multicolumn{2}{|l|}{ No. of Brain Metastases } & \multirow{4}{*}{0.84} \\
\hline $1(54)$ & $4.8(3.5-6.0)$ & \\
\hline 2-3 (41) & $3.4(1.1-5.7)$ & \\
\hline$>3(92)$ & $3.4(1.4-5.5)$ & \\
\hline \multicolumn{2}{|l|}{ ECM } & \multirow{3}{*}{0.37} \\
\hline Present (127) & $4.1(2.3-5.9)$ & \\
\hline Absent (89) & $2.7(1.0-4.4)$ & \\
\hline \multicolumn{2}{|l|}{ Systemic therapy } & \multirow{3}{*}{0.1} \\
\hline Yes (131) & $4.3(2.8-5.8)$ & \\
\hline No (42) & $2.8(1.4-4.1)$ & \\
\hline \multicolumn{2}{|l|}{ Primary } & \multirow{3}{*}{0.01} \\
\hline Controlled (19) & $9.9(0.0-26.9)$ & \\
\hline Uncontrolled (202) & $3.1(2.2-4.1)$ & \\
\hline
\end{tabular}

Table 4: Univariate analysis of median Overall survival (OS)

Various prognostic factors have been used either individually, or as a part of scoring system, to divide the patients into appropriate treatment groups. It is however not necessary that the prognostic factors extrapolated from one study population may be valid for all patients worldwide. Similar to our previous study, age and gender were not significant prognostic factors for OS [5]. This could be due to the fact that in the RTOG study population $47 \%$ patients were elderly and belonged to $>60$ year group whereas only $30 \%$ patients were $>60$ years in our study. This difference in age distribution may have affected the level of significance. Using different values for age grouping, based on local lung cancer population wise data, may be adopted in future for better correlation.

Adenocarcinoma histology has significantly better prognosis than non-adenocarcinoma for local control. However, very limited data is available showing significant differences in OS based on histology for lung cancer patients with BM. In this study, patients with adenocarcinoma histology had a significantly better OS $(p=0.01)$ as compared to those with non-adenocarcinoma histology. Sixty-four percent of these patients (106/165) received systemic therapy and 21 patients were EGFR positive. The benefit of having a favorable histology also extends to those patients who develop brain metastasis where it positively impacts the OS.

Number of brain lesion is also a significant prognostic factor for OS [8]. GPA score includes number of brain lesion as a prognostic factor whereas RPA does not. Number of brain metastasis however was not significant on univariate analysis for OS in our study. It has been shown that patients who undergo surgery followed by RT for oligo metastases have a sustained local control and better OS as compared to those offered only RT [9]. In our study 55 patients (25\%) presented with a single lesion but only 10 (18\%) underwent surgery for BM. Uncontrolled primary, poor KPS and patient refusal, were the reasons that contributed towards the low numbers undergoing surgery.

Extra cranial metastasis is a prognostic factor used by both RPA and GPA scoring, however in present study it did not emerge as a significant prognostic factor. Fifty-seven percent of patients had ECM in our study whereas fewer patients (27-46\%) had ECM in various RTOG studies [7]. The difference in the patient and tumor characteristics could be the reason for this.

RPA has been validated previously in our patient population and this study also confirms same in a larger cohort [5]. GPA score has been validated as a prognostic factor in some studies [1]. Multivariate analysis suggested GPA scoring as a significant prognostic factor for OS, however the difference was not significant across all groups. There

\begin{tabular}{|c|c|c|c|}
\hline Variable & p-Value & HR & Cl \\
\hline \multicolumn{4}{|c|}{ Presentation } \\
\hline Synchronous & 0.01 & 0.62 & $0.46-0.85$ \\
\hline Metachronous & \multicolumn{4}{|c|}{ Histology } \\
\hline \multicolumn{4}{|c|}{ RPA } \\
\hline Adeno & 0.01 & 0.65 & $0.46-0.91$ \\
\hline Non-Adeno & 0.05 & 0.72 & $0.52-1.00$ \\
\hline & \multicolumn{4}{|c|}{1} \\
\hline I-II & GPA Score & 1 & \\
\hline III & 0.05 & 0.23 & $0.05-1.00$ \\
\hline $3.5-4.0$ & 0.19 & 0.67 & $0.37-1.21$ \\
\hline 3 & 0.51 & 0.89 & $0.64-1.24$ \\
\hline $1.5-2.5$ & & 1 & \\
\hline $0-1$ & & & \\
\hline
\end{tabular}

Table 5: Multivariate analysis of median overall survival.

\begin{tabular}{|c|c|c|c|}
\hline \multicolumn{5}{|c|}{ Gaspar et al. [6] } & Sperduto et al. [7] & Present study \\
\hline \multicolumn{5}{|c|}{ RPA Class } \\
\hline II & 7.1 & 7.7 & 8.8 \\
\hline III & 4.2 & 4.5 & 5.2 \\
\hline \multicolumn{4}{|c|}{ GPA } \\
\hline $0-1$ & 2.3 & 2.3 & 2.6 \\
\hline $1.5-2.5$ & - & 2.6 & 2.4 \\
\hline 3 & - & 3.6 & 4 \\
\hline $3.5-4.0$ & - & 6.9 & 4.8 \\
\hline
\end{tabular}

Table 6: Median OS in months for Brain Metastases. 
Citation: Tibdewal AR, Pathak RS, Laskar SG, Agarwal JP (2016) Prognostic Factors and Survival Outcomes of Lung Cancer Patients with Brain Metastasis: Experience of a Tertiary Cancer Centre. J Brain Tumors Neurooncol 1: 106. doi: 10.4172/2475-3203.1000106

Page 5 of 5

was a significant difference ( $\mathrm{p}=0.05$ )in OS for patients with GPA score 3.5-4.0 when compared with GPA of 0-1.0 (Table 5). This could be explained by the fact that age, ECM and number of brain lesion were not found to be significant prognostic factors for our study population. Inclusion of statistically insignificant factor(s) as a part of prognostic grouping affects the overall scoring system making it in-effective in dividing patient population according to their survival end point. However, the overall difference between the two extreme groups was too large to not be detectable and hence was significant on multivariate analysis.

Time of presentation of BM at diagnosis or after treatment at follow up also impacts the outcome [3,4]. In our study we found that patients with synchronous BM (detected within 2 months of primary diagnosis) have good prognosis as compared to those with metachronous BM. A similar study evaluating outcomes of solitary BM patients undergoing treatment with Gamma knife stereotactic radio surgery, however, found no significant survival difference between synchronous and metachronous presentation [3]. Superior outcome in patients with synchronous BM ( $\mathrm{p}$ value not significant) as compared to metachronous $\mathrm{BM}$ in our patient population could be attributed to administration of 1st line chemotherapy/ targeted therapy in systemictreatment naïve patients. Patients with synchronous metastasis gain benefit with systemic therapy when instituted early whereas patients with metachronous $\mathrm{BM}$ are already pre-treated most often with 1st or second line systemic therapy and would have either progressed on treatment or soon after. The cancer cells develop resistance to drugs used which make them less responsive to further lines of systemic therapies ultimately resulting in poorer survival. When synchronous BM patients were excluded from the analysis, we did not find any significant difference in OS between patients with disease free interval (DFI) of 12 months or more. However, a study by Rades et al. showed superior OS with $>12$ month DFI when compared with $<12$ months DFI [10].

Addition of systemic treatment in the form of chemotherapy and/ targeted therapy did not significantly improve OS in our subset of patients $(p=0.1)$ as compared to those that did not receive any further systemic treatment. Sixty percent patients received systemic therapy after completion of WBRT and remaining patients were either not eligible for systemic therapy or failed to report to the clinic after WBRT completion for further therapy.

One fourth of our patients belonged to the 30-day mortality group. In this study $19 / 115$ (16.5\%) patients with favourable RPA class I-II and $8 / 22(36.4 \%)$ patients with GPA score of $\geq 2.5$ died within 30 days of their diagnosis. Whereas, the group of RPA class III and GPA score of 0-1.0 with poorest prognosis had median OS of 2.3 and 2.6 months respectively. This retrospective study suggests that RPA class and GPA score failed to accurately identify patients within the 30 day mortality group. It is indeed important to be able to identify these patients to prevent them from being subjected to ineffective protracted treatments in their last few days of life. The focus should however be on providing them with best supportive care to ease their suffering. This would also help in channelizing RT resources appropriately in a resource constrained setting.
The limitations of our study were its retrospective nature, lack of documentation of neurocognitive outcomes and QOL for these patients which are important surrogate outcome measure for patients with poor OS. Incorporating the salient features of this study in routine clinical practice will help to tailor the treatment of patients with BM. Further prospective studies are underway at our institution which addresses the limitations of this study.

\section{Conclusions}

Ideal scoring system should be able to accurately prognosticate survival and thereby aid in appropriate institution of therapy. GPA scoring could be validated only for accurately predicting OS of patients of lung cancer with BM having the best and the poorest survival in Indian population. A quarter of our patients died within 30 days of diagnosis of $\mathrm{BM}$ and most prognostic variables failed to determine the brevity of their life. BM patients are a heterogeneous group and further distinction in grouping by incorporation of molecular markers may be considered.

\section{Reference}

1. Sperduto PW, Chao ST, Sneed PK, Luo X, Suh J, et al. (2010) Diagnosisspecific prognostic factors, indexes, and treatment outcomes for patients with newly diagnosed brain metastases: a multi-institutional analysis of 4,259 patients. Int J Radiat Oncol Biol Phys 77: 655-661.

2. Sperduto PW, Kased N, Roberge D, Xu Z, Shanley R, et al. (2012) Summary report on the graded prognostic assessment: an accurate and facile diagnosisspecific tool to estimate survival for patients with brain metastases. J Clin Oncol 30: 419-425.

3. Flannery TW, Suntharalingam M, Kwok Y, Koffman BH, Amin PP, et al. (2003) Gamma knife stereotactic radiosurgery for synchronous versus metachronous solitary brain metastases from non-small cell lung cancer. Lung cancer 42: 327 333.

4. Ali A, Goffin JR, Arnold A, Ellis PM (2013) Survival of patients with non-smallcell lung cancer after a diagnosis of brain metastases. Curr Oncol 20: e300306.

5. Agarwal JP, Wadasadawala T, Munshi A, Chadda P, Apsani R, et al. (2010) Validation of Recursive Partitioning Analysis Classification in Patients with Brain Metastases from Non-small Cell Lung Cancer Treated with Short-course Accelerated Radiotherapy. Clin Oncol 22: 837-843.

6. Gaspar L, Scott C, Rotman M, Asbell S, Phillips T, et al. (1997) Recursive partitioning analysis (RPA) of prognostic factors in three Radiation Therapy Oncology Group (RTOG) brain metastases trials. Int J Radiat Oncol Biol Phys 37: 745-751.

7. Sperduto PW, Berkey B, Gaspar LE, Mehta M, Curran W (2008) A new prognostic index and comparison to three other indices for patients with brain metastases: an analysis of 1,960 patients in the RTOG database. Int J Radiat Oncol Biol Phys 70: 510-514.

8. Andrews DW, Scott CB, Sperduto PW, Flanders AE, Gaspar LE, et al. (2004) Whole brain radiation therapy with or without stereotactic radiosurgery boost for patients with one to three brain metastases: phase III results of the RTOG 9508 randomised trial. Lancet 363: 1665-1672.

9. Patchell RA, Tibbs PA, Walsh JW, Dempsey RJ, Maruyama Y, et al. (1990) A randomized trial of surgery in the treatment of single metastases to the brain. N Engl J Med 322: 494-500.

10. Rades D, Schild SE, Lohynska R, Veninga T, Stalpers LJA, et al. (2007) Two Radiation Regimens and Prognostic Factors for Brain Metastases in Nonsmall Cell Lung Cancer Patients. Cancer 110: 1077-1082. 